\title{
Novel Feature Extraction Methodology with Evaluation in Artificial Neural Networks Based Fingerprint Recognition System
}

\author{
Nihan KAHRAMAN, Zehra Gulru CAM TASKIRAN, Murat TASKIRAN
}

\begin{abstract}
Fingerprint recognition is one of the most common biometric recognition systems that includes feature extraction and decision modules. In this work, these modules are achieved via artificial neural networks and image processing operations. The aim of the work is to define a new method that requires less computational load and storage capacity, can be an alternative to existing methods, has high fault tolerance, convenient for fraud measures, and is suitable for development. In order to extract the feature points called minutia points of each fingerprint sample, Multilayer Perceptron algorithm is used. Furthermore, the center of the fingerprint is also determined using an improved orientation map. The proposed method gives approximate position information of minutiae points with respect to the core point using a fairly simple, orientation map-based method that provides ease of operation, but with the use of artificial neurons with high fault tolerance, this method has been turned to an advantage. After feature extraction, General Regression Neural Network is used for identification. The system algorithm is evaluated in UPEK and FVC2000 database. The accuracies without rejection of bad images for the database are $95.57 \%$ and $91.38 \%$ for UPEK and FVC2000 respectively.
\end{abstract}

Keywords: Artificial Intelligence; Feature Extraction; Fingerprint Recognition; Neural Networks

\section{INTRODUCTION}

Human fingerprints are surrounded by ridges and valleys which together outline unique patterns for every individual. These patterns are formed from the beginning of life and are permanent through the whole lifetime. However, damages on fingerprint like cuts or burns may change the quality of it. Various studies in the literature examined that two people even twins do not have the same fingerprints. This is one of the most important features of biometrics, called uniqueness [1]. As biometrics measurements, fingerprints are used due to these properties in many works including law enforcements such as crime, forensics, border security etc. [2-4]. Furthermore, there are many products that use automatic fingerprint recognition systems such as mobile phones, computers or cash dispensers equipped with fingerprint sensing devices $[5,6]$.

Although a lot of algorithms have been developed for creating new automatic fingerprint recognition systems since 1960s, fingerprint matching is still the most important problem for these systems. It is still a challenging task to improve algorithms that gives better solutions to Automatic Fingerprint Recognition Systems (AFIS). They appear as a low-cost and low-power alternative to traditional person identification systems because of inexpensive fingerprint readers with high performance processor technology [1]. Furthermore one recent development is secure key generation algorithms using biometric cryptosystems. In such systems, the security levels used as the advantage of cryptography and the irrefutability of biometric data are combined to eliminate the need to carry any markers. Because of increasing attention shown to neural networks application in solving real world problems, fingerprint recognition systems will include these applications at least as a part. Here, recognition includes both identification and verification in biometrics [1]. There is a penetrating difference between them. Verification systems generally use matching methods to compare presented fingerprint with enrolled database on an individual basis one by one and in each trial they give a similarity score between two fingerprints. On the other hand, identification systems search a biometric to identify an unknown user. Generally, fingerprint identification systems are based on a minutiae extraction method.

Neural networks [7, 8], fuzzy logic [9], Gabor filters [10], line tracing algorithms $[11,13]$, orientation based methods [14] and simple convolutional filters [15] are some of the common methods to extract the minutiae points. However, line tracing algorithms and convolutional filters are time consuming. Furthermore, many fingerprint recognition systems in the literature including neural networks give the results of verification instead of identification. Neural networks can be used in fingerprint recognition systems in two stages. More usual one is to use neural networks in pattern recognition stage $[9,10,14,15]$. However there are also some works that use neural networks in feature extraction stage $[7,11,12]$.

Fingerprint recognition systems start with observing many details called minutiae in form of discontinuities in ridges. Another important point is that a minutia does not change over time. Basic minutiae points that are found in general fingerprints can be seen in Fig 1.

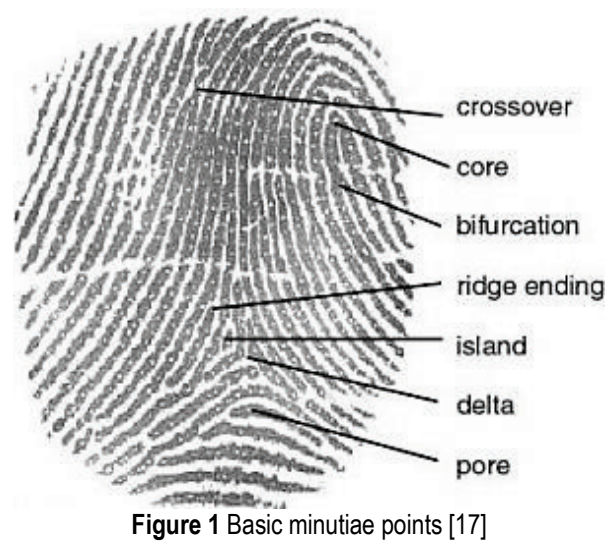

Here, the dark lines represent the ridges while the light lines represent the valleys. After detailed examination, the two most important minutiae are preferred as the ridge end and the bifurcation. The 
remainders are only the combination of these two types of minutiae. The minutiae mapping algorithm is a good way to determine whether two fingerprints belong to the same person (verification). Furthermore minutia matching can also be used in identification. However, the reliability of the minutiae features depends on image acquisition method and discontinuities in the image directly affect the identifier performance. In this study, completer orientation filter is used to increase the minutiae extraction and matching performance.

The core point detection is based on the orientation field. Most common core finding method is benefitted from the orientation of all ridges and chose the $U$ turn point as core $[17,18]$, but these methods may be unsuccessful where the database includes deficient information on some fingerprints. To find the core point, a unique method based on simple circle fitting process is applied. This proposed alternative method has the advantage of not to need whole fingerprint image. A fingerprint image is satisfactory for the core point coordinate detection with high approximation rate to have two angle fields area. Therefore, this feature directly affects positively the system performance. The paper first explains novelties in feature extraction steps in Section II including core point detection method and Section III describes recognition via General Regression Neural Networks (GRNN). Conclusion part includes comparisons between proposed method results and literature

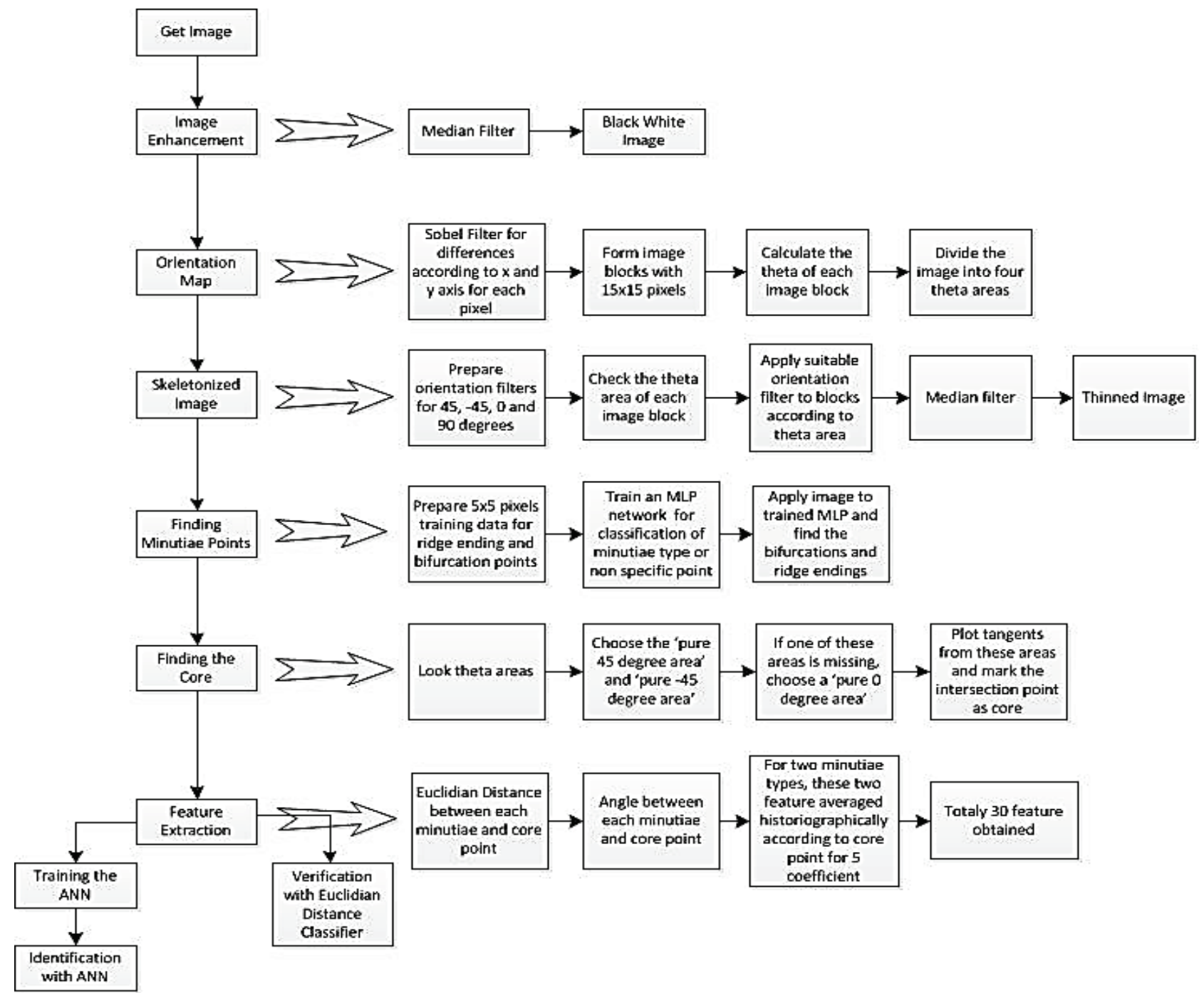

Figure 2 Block diagram of Fingerprint Classification Algorithm

\section{PROPOSED FINGERPRINT FEATURE EXTRACTION METHODOLOGY}

Fingerprint recognition, which is a quick and easy solution to security systems, has become one of the most widely used methods to date. There are various studies on fingerprint recognition systems including novelties and successful accuracies $[2,19,20]$. This paper also proposes a new methodology for fingerprint recognition systems. Fig. 2 presents the proposed framework for fingerprint recognition system. The proposed flow chart includes image enhancement, orientation map, skeletonized image, finding minutiae points, finding the core, feature extraction and classification steps. Right side of Fig. 2 shows the methodologies used in these steps.

As seen from Fig. 2, morphological operations and filtering should be evaluated on a fingerprint image in order to enhance the information before feature extraction steps. Removing the noise and making binarization are the first two steps to provide the image to the recognition 
system. $3 \times 3$ Median filter is applied to eliminate the corruptive effect of noise, and converting grey scale image into binary image by fixing the threshold value so that the image is prepared for subsequent processes. Original image and binary image for a sample fingerprint can be seen in Fig. 3.

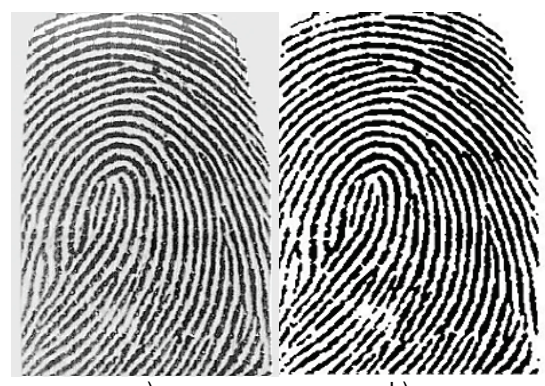

a)

Figure 3 a) Original fingerprint image and b) fingerprint after binarization

Minutia extraction and matching depend heavily on the correct estimation of local ridge orientation. Therefore, after enhancement techniques, in order to determine all local orientation angles on the image, named as forming orientation map, $3 \times 3$ Sobel filter is used [26]. Some other researchers proposed different methodologies such as orientation estimation based on gradients computation [21-25]. Mathematically, Sobel filter uses two $3 \times 3$ kernels which are convolved with the original image to calculate the derivatives. One of these kernels is used for horizontal changes while the other for vertical changes. Let $\boldsymbol{G}_{x}$ and $\boldsymbol{G}_{y}$ be horizontal and vertical derivatives and $\boldsymbol{A}$ is the fingerprint image. Oriented gradients can be calculated as in Eq. (1) and Eq. (2) where * denotes the convolution operator.

$\boldsymbol{G}_{x}=\left[\begin{array}{ccc}1 & 0 & -1 \\ 2 & 0 & -2 \\ 1 & 0 & -1\end{array}\right] * \boldsymbol{A}$,

$\boldsymbol{G}_{y}=\left[\begin{array}{ccc}1 & 2 & 1 \\ 0 & 0 & 0 \\ -1 & -2 & -1\end{array}\right] * \boldsymbol{A}$.

Using these two derivatives, directions of all pixels denoted by $\theta$, can be calculated as in Eq. (3).

$\theta=\tan ^{-1}\left(\frac{\boldsymbol{G}_{y}}{\boldsymbol{G}_{x}}\right)$.

In order to form the orientation map, image is partitioned into $15 \times 15$ pixel sized grids and the orientation filter, which is shown in Tab. 1, is applied to the grids for $0^{\circ}$. The mean value of angles in each grid is chosen as orientation of the block. In this way, orientation map is reformed by rounding the angle of each block to the closest angle into four angle fields which are $0^{\circ}, 45^{\circ}$, $90^{\circ}$ and $-45^{\circ}$. Thinning is the next process for reducing thickness of each line of patterns into just a single pixel width. However, the thinned fingerprint image should be one single pixel width with no discontinuities which can

be originated from scanning device or threshold error during binarization process. Orientation filters can accomplish these types of faults. In this work, four orientation filters were applied to thinned fingerprint image [27]. Each filter determines a direction vector horizontal, vertical, or $\pm 45^{\circ}$. According to block angle field, the proper orientation filter is applied to each $15 \times 15$ image block and smoother image is obtained without discontinuities. A sample filter used for $0^{\circ}$ orientation is given in Tab. 1.

Table 1 Used Orientation filter for $0^{\circ}$

\begin{tabular}{|l|l|l|l|l|l|l|l|l|l|l|}
\hline-115 & -226 & -323 & -396 & -443 & -459 & -443 & -396 & -323 & -226 & -115 \\
\hline-106 & -209 & -298 & -366 & -409 & -424 & -409 & -366 & -298 & -209 & -106 \\
\hline-81 & -160 & -228 & -280 & -313 & -324 & -313 & -280 & -228 & -160 & -81 \\
\hline 0 & 0 & 0 & 0 & 0 & 0 & 0 & 0 & 0 & 0 & 0 \\
\hline 177 & 349 & 497 & 611 & 683 & 707 & 683 & 611 & 497 & 349 & 177 \\
\hline 250 & 494 & 703 & 864 & 965 & 1000 & 965 & 864 & 703 & 494 & 250 \\
\hline 177 & 349 & 497 & 611 & 683 & 707 & 683 & 611 & 497 & 349 & 177 \\
\hline 0 & 0 & 0 & 0 & 0 & 0 & 0 & 0 & 0 & 0 & 0 \\
\hline-81 & -160 & -228 & -280 & -313 & -324 & -313 & -280 & -228 & -160 & -81 \\
\hline-106 & -209 & -298 & -366 & -409 & -424 & -409 & -366 & -298 & -209 & -106 \\
\hline-115 & -226 & -323 & -396 & -443 & -459 & -443 & -396 & -323 & -226 & -115 \\
\hline
\end{tabular}

After the orientation filter, the ridges in the image are stretched through the orientations. To remove these false ridges, a mask covering the finger bounds should be applied to the image. The boundary of mask is determined with a suitable threshold level applied to the grey scale image. To obtain a sharp mask, the spots in the fingerprint region are removed with a $3 \times 3$ median filter. We have discarded outlier endings by applying an 'AND' process between mask and binary image. Thinning process is evaluated with high accuracy after masking process. Thinning does not change the location and orientation of minutiae points compared to original fingerprint which ensures accurate estimation of minutiae points. Output of masked orientation filter and thinned image can be seen in Fig. 4.

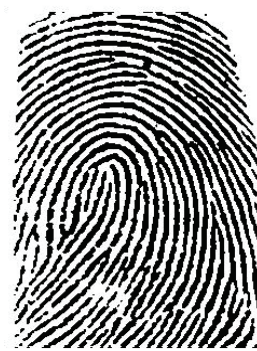

a)

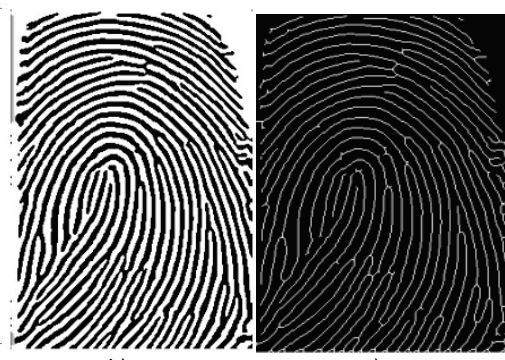

b)

c)
Figure 4 a) Original fingerprint image, b) Output of orientation filter after masking, c) thinned fingerprint image

\subsection{Training Artificial Bee Colony-MLP Hybrid Architecture for Minutiae Extraction in Fingerprint}

In this work, a minutia extraction method based on artificial neural network is used. As mentioned in Introduction part, the most frequently used minutiae points are ridge ends and bifurcation points. These minutiae points are introduced to a Multi-Layer Perceptron by including all possible $5 \times 5$ pixel matrices. Fig. 6 presents a sample of $5 \times 5$ pixel areas for both ridge ending and bifurcation respectively. In this frame, 100 matrix samples which contain bifurcation points, ridge 
ends and not-a-minutiae-point, are applied to 25-input, 2output MLP network as training dataset (Fig. 5).

\begin{tabular}{|c|c|c|c|c|}
\hline 0 & 0 & 0 & 0 & 0 \\
\hline 0 & 0 & 0 & 0 & 0 \\
\hline 1 & 1 & 1 & 0 & 0 \\
\hline 0 & 0 & 0 & 0 & 0 \\
\hline 0 & 0 & 0 & 0 & 0 \\
\hline
\end{tabular}

\begin{tabular}{|c|c|c|c|c|}
\hline 0 & 0 & 0 & 0 & 1 \\
\hline 0 & 0 & 0 & 1 & 0 \\
\hline 1 & 1 & 1 & 0 & 0 \\
\hline 0 & 0 & 0 & 1 & 0 \\
\hline 0 & 0 & 0 & 0 & 1 \\
\hline
\end{tabular}

Figure 5 Sample ridge end and bifurcation training matrices

Since the activation function, learning speed, momentum coefficient and hidden neuron numbers used in the training of multilayer perceptron networks are generally determined by trial and error method, determination of the optimal network structure leads to an increase in the training period. Learning rate and momentum coefficient are critical parameters in order to ensure that the learning process does not stick to a local optimum point. Furthermore, hidden neuron number has an effect on classification accuracy where excessive number of hidden neuron causes an increase of the operation time. Activation function type directly determines the MLP performance. In this work, these four parameters are chosen with Artificial Bee Colony (ABC) [28] optimization algorithm. ABC system conducts a balanced process between exploration and exploitation by using employed and onlooker bees for local search and, scouts and onlooker bees for global search. In this work, half of the bees in colony are selected as employed bee and remaining are onlookers in $\mathrm{ABC}$ Algorithm for this problem. Limit parameter in $\mathrm{ABC}$ is determined as 10 . The count of iteration that gives the best result is noted. The final solution is considered to be the best solution when all solutions stop improving. MLP classifier is operationalized by using this Back Propagation-Artificial Bee Colony hybrid training method. For optimum network topology, a multilayer perceptron with one hidden layer is designed. Initial weights are selected randomly. Input neuron number is 25 from the pixel number of $5 \times 5$ training matrices. The hidden neuron number in the hidden layer is determined 32 from the result of optimization algorithm. Sigmoid function is also chosen by optimization as activation function. Other optimization results used in training process are 0.6453 for learning rate and 0.4892 for momentum coefficient.

\subsection{Detection of Core Point in Fingerprint}

Conventional detection algorithms for core point use the orientation of all ridges and choose the $U$ turn point as core $[17,18]$. However they may be unsuccessful where the database includes deficient information on some fingerprints. In this work, in order to find the core point, the orientation map is used in the first step. Pure $45^{\circ}$ fields and pure $-45^{\circ}$ fields which have the area of at least 3 complete blocks are searched. In Fig. 6, red marks and blue marks show pure $45^{\circ}$ and pure $-45^{\circ}$ fields respectively. If one of these fields isn't found, pure $0^{\circ}$ field is searched instead of the missing field. The smoothest ridge can be determined by its length of at least
20 pixels with discontinuities. For both $45^{\circ}$ and $-45^{\circ}$ fields, tangents of these smoothest lines are intercepted. This intercept point is marked as core point (the green mark) as can be seen in Fig. 8. This methodology is one of the major novelties in this paper.

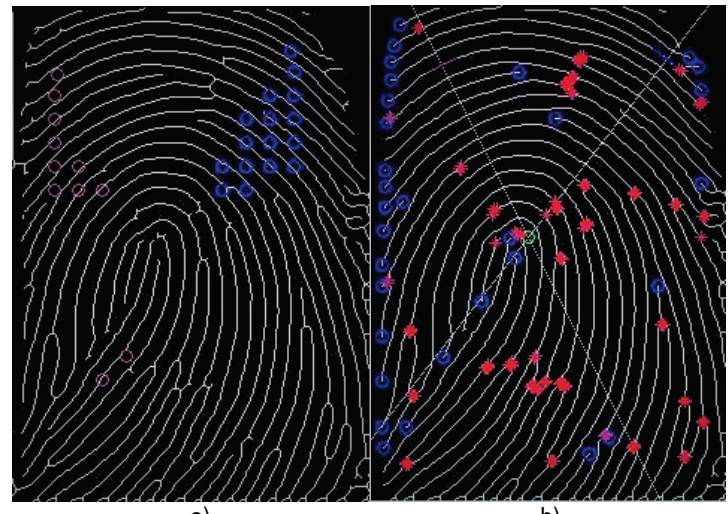

a)

b)

Figure 6 a) Pure $45^{\circ}$ fields with red circles and $-45^{\circ}$ fields with blue circles; $b$ ) Core point with green mark, bifurcation points with red mark and ridge ends with blue mark

\subsection{Feature Vector Extraction}

The type, angle according to core point, distance along the $\mathrm{x}$ and $\mathrm{y}$ axis of each minutiae point are determined as features to identify the fingerprint images. After detecting core point, features are extracted using histogram of minutia, which is a contribution to the literature. First the nearest and the extreme minutiae points are specified. The distance between these two minutiae points is divided horizontally into five regions. Then histogram of minutiae in the $y$ axis for each region is obtained. Same procedure is applied vertically and azimuthally so that histogram of minutiae in the $x$ axis and in angular value are obtained respectively. Therefore histograms of minutia points are evaluated in a special region of interest, and these histogram and the angles are used as feature vector. This methodology is also applied to both bifurcations and ridge endings separately. Totally 30 features are obtained including;

- Number of $x$ values of endings in each region,

- Number of $y$ values of endings in each region,

- Section of angular areas of endings in each region,

- Number of $x$ values of bifurcations in each region,

- Number of $y$ values of bifurcations in each region,

- Section of angular areas of bifurcations in each region.

This feature vector is applied as input to Generalized Regression Neural Network [29] (GRNN) in order to decide the identity of the fingerprint image. GRNN is a variation of the radial basis neural networks, which is based on kernel regression networks. This memory-based network converges to a linear or non-linear surface with the estimation of continuous variables. It has a highly parallel structure. It is quite successful in solving regression problems and does not need linear assumptions. Thanks to its parallel structure, it does not require an iterative training procedure like other methods such as back propagation algorithm. This is an advantage because the estimated values cannot converge to poor 
solutions corresponding to local minimum of the error criterion.

It basically consists of four basic blocks: input units, pattern units, summation units and output units. The number of input units in input layer is the total number of observed parameters. The outputs from the first layer are transferred to the pattern unit where each neuron represents a training pattern and its output. In the training part of these neurons, radial based and linear activation functions are used. In the summation unit following these layers, two different types of summation are executed in parallel, which are a single division unit and summation units. One of them ( $S$-neurons) computes the weighted sums and the other one ( $D$-neurons) use un-weighted outputs of pattern unit. Through the collaboration of the last two layers, a normalized output set is obtained. The output layer only divides the output of each $S$-summation neuron by that of each $D$-summation neuron, yielding the predicted value $Y_{i}$ to an unknown input vector $x$ as,

$$
Y_{i}^{\prime}=\frac{\sum_{i=1}^{n} y_{i} \cdot \exp -D\left(x, x_{i}\right)}{\sum_{i=1}^{n} \exp -D\left(x, x_{i}\right)},
$$

$$
D\left(x, x_{i}\right)=\sum_{k=1}^{m}\left(\frac{x_{i}-x_{i k}}{r}\right)^{2} .
$$

$y_{i}$ - weight connection between the ith neuron in the pattern layer and the $S$-summation neuron,

$n$ - number of the training patterns,

$D$ - Gaussian function, $m$ is the number of elements of an input vector,

$x_{i k}-k^{\text {th }}$ element of $x_{i}$, respectively,

$r$ - spread parameter, whose optimal value is determined experimentally [30].

In this work, GRNN network has 30 inputs and 4 outputs (individual identity). Proposed method is tested with two datasets. First, the fingerprint database of UPEK Inc. (one of the global leaders in biometric fingerprint security solutions) contains totally 128 images collected from 16 individuals. Fingerprint Verification Competition (FVC) in 2000 [31] is used as second dataset. It contains 80 samples of image collected from 10 individuals. According to 4 -fold cross correlation, $75 \%$ of both two datasets are used as training data and the remaining is used as testing data.

\section{FINGERPRINT RECOGNITION RESULTS USING PROPOSED METHODOLOGY}

In this work histogram of minutiae based recognition system is tested with two datasets. Both of these datasets have deficient samples even not including core point of a fingerprint. Therefore we have proposed a new methodology for defining the coordinates of core point. The novelty is explained in Section 2.2 and extracted feature vector. The test results in this paper include both verification and identification on datasets mentioned in Section 2. So many studies in literature give accuracies for UPEK and FVC2000 databases, however it is unclear that recognition includes identification or verification.

\subsection{UPEK Dataset Verification Results}

This dataset consists of 16 individuals and 8 fingerprints for each, totally 128 samples. In order to obtain False Accept Rate (FAR) and False Reject Rate (FRR) results, each sample is compared to others using Euclidian distances. This distance matrix is $128 \times 128$ and has 16384 data, however this matrix is symmetrical and therefore we considered half of it. After choosing threshold as Euclidian distance 0.3 between each feature vector, we obtained "Genuine Matrix". Finally, 7829 samples over 8192 , which means $95.57 \%$, are positively verified with exact individual, where $F A R=0.0137$ and $\mathrm{FRR}=0.5039$.

Table 2 FAR, FRR and Accuracy results for different threshold values of Euclidian distances between individual's feature vectors in UPEK dataset

\begin{tabular}{|c|c|c|c|}
\hline $\begin{array}{c}\text { UPEK } \\
\text { (Threshold) }\end{array}$ & FAR & FRR & $\begin{array}{c}\text { Test Accuracy } \\
\%\end{array}$ \\
\hline 0 & 0 & 0.8750 & 94.53 \\
\hline 0.1 & 0 & 0.8750 & 94.53 \\
\hline 0.2 & 0.00026 & 0.7813 & 95.09 \\
\hline 0.3 & 0.0137 & 0.5039 & 95.57 \\
\hline 0.4 & 0.1104 & 0.2324 & 88.20 \\
\hline 0.5 & 0.3043 & 0.0830 & 70.95 \\
\hline 0.6 & 0.5479 & 0.0293 & 48.45 \\
\hline 0.7 & 0.7586 & 0.0020 & 28.87 \\
\hline 0.8 & 0.8977 & 0 & 15.84 \\
\hline 0.9 & 0.9871 & 0 & 7.46 \\
\hline 1 & 1 & 0 & 6.25 \\
\hline
\end{tabular}

Tab. 2 shows FAR and FRR results due to choosing threshold as distance between individual's feature vectors and Fig. 7 shows FAR-FRR distribution graph.

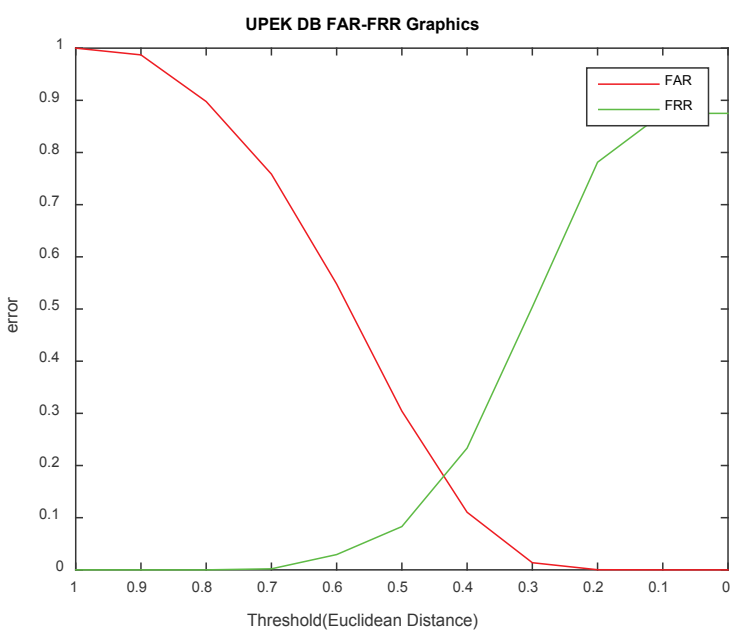

Figure 7 FAR-FRR distribution graph for UPEK fingerprint dataset

\subsection{UPEK Dataset Identification Results}

Fingerprint identification is the method of identifying an individual through the others. Since no two individuals have exactly the same layout of ridge patterns, and the patterns of any individual remain unchanged throughout life, fingerprints offer an infallible biometric by means of personal identification. Neural network classifiers are commonly used in biometric recognition systems due to their high tolerance to noisy data [12]. In this paper, four 
different types of artificial neural networks are used for classification: Multi-Layer Perceptron (MLP), Radial Basis Function Neural Networks (RBF), Probabilistic Neural Networks (PNN) and Generalized Regression Neural Networks (GRNN). Usually GRNN has shown superiority over the MLP because in GRNN the training may be in terms of global or local basis functions whereas MLP applies a global basic function.

The GRNN is based on the estimation of probability density functions, and RBF is based on iterative function approximation. Although PNN and GRNN are based on the estimation of probability density functions, the reason that GRNN and RBF outperform compared with PNN, could be the use of the regression method. Tab. 3 shows the approximate results for the UPEC dataset classification. The best accuracy is acquired with GRNN classification after proposed feature extraction methods.

Table 3 Comparison of ANNs classification accuracies in UPEK dataset

\begin{tabular}{|c|c|c|c|c|}
\hline & PNN & RBF & MLP & GRNN \\
\hline Accuracy \% & 25 & 68.75 & 78.13 & $\mathbf{9 5 . 5 7}$ \\
\hline
\end{tabular}

\subsection{FVC2000 DB2 Dataset Verification Results}

This dataset consists of totally 6400 samples. FAR $=$ 0.0358 and $\mathrm{FRR}=0.5750$ are calculated using optimum threshold value as explained in Section 3.1. Tab. 4 shows FAR and FRR results due to choosing threshold as distance between individual feature vectors and Fig. 8 shows FAR-FRR distribution graph.

Table 4 FAR, FRR and Accuracy results for different threshold values of Euclidian distances between individual's feature vectors in FVC2000 DB2

\begin{tabular}{|c|c|c|c|}
\hline \multicolumn{5}{|c|}{ dataset } \\
$\begin{array}{c}\text { FVC } \\
\text { (Threshold) }\end{array}$ & FAR & FRR & $\begin{array}{c}\text { Test Accuracy } \\
\%\end{array}$ \\
\hline 0 & 0 & 0.8750 & 91.25 \\
\hline 0.1 & 0.00034 & 0.8594 & 91.38 \\
\hline 0.2 & 0.0358 & 0.5750 & 91.03 \\
\hline 0.3 & 0.2146 & 0.1906 & 78.78 \\
\hline 0.4 & 0.5243 & 0.1156 & 51.66 \\
\hline 0.5 & 0.7503 & 0.0781 & 31.69 \\
\hline 0.6 & 0.8503 & 0.0375 & 23.09 \\
\hline 0.7 & 0.9156 & 0.0344 & 17.25 \\
\hline 0.8 & 0.9503 & 0.0063 & 14.41 \\
\hline 0.9 & 0.9875 & 0 & 11.12 \\
\hline 1 & 0.9997 & 0 & 10.03 \\
\hline
\end{tabular}

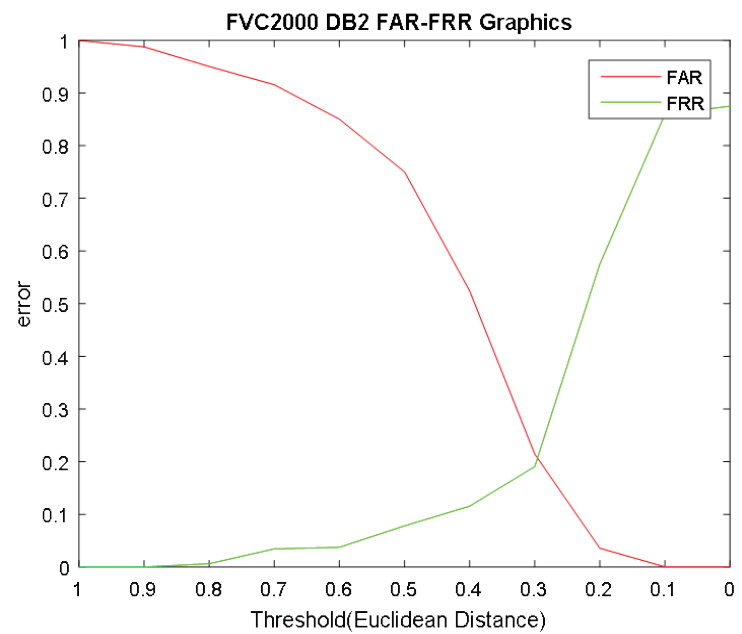

Figure 8 FAR-FRR distribution graph for FVC2000DB2 fingerprint dataset

\subsection{FVC2000 DB2 Dataset Identification Results}

Above mentioned ANN algorithms were also used for classification of FVC2000DB2 dataset and we have again obtained the best accuracy with GRNN classification as shown in Tab. 5 after proposed feature extraction methods.

Table 5 Comparison of ANNs classification accuracies in FVC2000DB2 dataset

\begin{tabular}{|c|c|c|c|c|}
\hline & PNN & RBF & MLP & GRNN \\
\hline Accuracy \% & 39.86 & 31.1 & 80.7 & $\mathbf{9 1 . 3 8}$ \\
\hline
\end{tabular}

\section{CONCLUSION}

In order to determine or verify the identity of an individual, nowadays biometric systems including fingerprint, palm print, face, iris, retina, ear, voice, signature, gait, hand vein, odor, or the DNA information are used. In this study, a histogram-based, error-tolerant method is proposed as an alternative to the successful methods available for fingerprint recognition. In the proposed method, first, noise in a fingerprint image is reduced by morphological operations. Here the feature extraction methodology on minutiae points, end points and bifurcations, include novel steps. The histogram of distance between minutiae points and core point, and angles between these characteristics are given as input to the neural network. The histogram of minutiae based recognition system is tested with two datasets. Both of these datasets have deficient samples even not including core point of a fingerprint. Thus a new methodology for defining the coordinates of core point is proposed. Though the obtained distance and angle information are approximate, the fault tolerance characteristics of neural networks are used as an advantage. This system is designed to tolerate the image orientation differences. Because of regression based approach of GRNN, high identification performance is obtained for both used datasets. In literature, high accuracy rates are obtained by rejecting some patterns which appeared to belong to the wrong classes after the classification process $[32,33]$. The proposed system algorithm is evaluated in UPEK and FVC2000 database without rejection. The accuracies for the database are $95.57 \%$ and $91.38 \%$ for UPEK and FVC2000 respectively. Although these results are more successful than some studies [34, 35], generally at the close level of success rates provided by accepted methods in the literature $[36,37,38]$. However, these accepted methods use direct matching techniques that requires high storage capacity and computational capacity because they use direct comparison methods [39], and thus they are not efficient in terms of time and cost $[36,37]$. In the study of Bei et al. [38], the results are not obtained by folded cross validation and it leads to lack of information about the success of artificial neural network. Because the most discriminating feature used to distinguish between synthetic and real fingerprints is the histograms of minutiae points [40], measures against this fraud can also be taken with the features used in this method. Test results show the effectiveness and reliability of this algorithm in terms of accuracy rates, and ease of implementation, fault tolerance, and structure suitable for development and measures against fraud show that the 
proposed method may be an alternative to classical methods.

\section{REFERENCES}

[1] Jain, A. K., A. Ross, A., \& Nandakumar, K. (2011). Introduction to Biometrics. ISBN 978-0-387-77326-1. https://doi.org/10.1007/978-0-387-77326-1

[2] Wang, G., Zhou, J., Yang, Z., \& Yang, X. (2011). Application of fractional-order differentials in enhancing vestige images of crime scene. $6^{\text {th }}$ IEEE Joint International Information Technology and Artificial Intelligence Conference (ITAIC), 1, 295-298. https://doi.org/10.1109/ITAIC.2011.6030209

[3] Alves, M. G., Speglich, C., Trevelin, L. C., \& ArajoMoreira, F. M. (2012). Bioinformatics applied to public safety: improving fingerprint revelation left in crime scenes to criminal identification in real time. IEEE International Conference on Systems, Man, and Cybernetics (SMC), 2306-2311. https://doi.org/10.1109/ICSMC.2012.6378085

[4] Prabhusundhar, P., Kumar, V. K. N., \& Srinivasan, B. (2013). Border crossing security and privacy in biometric passport using cryptographic authentication protocol. International Conference on Computer Communication and Informatics (ICCCI), 1-7. https://doi.org/10.1109/ICCCI.2013.6466144

[5] Qi, Z., Wen, W., Meng, W., Zhang, Y., \& Shi, L. (2014). An energy efficient OpenCL implementation of a fingerprint verification system on heterogeneous mobile device. IEEE $20^{\text {th }}$ International Conference on Embedded and Real-Time Computing Systems and Applications (RTCSA), 1-8.

[6] Kumar, D. \& Ryu Y. (2009). A brief introduction of biometrics and fingerprint payment technology. International Journal of Advanced Science and Technology, 4, 25-37.

[7] Montesanto, A., Baldassarri, P., Vallesi, G., \& Tascini, G. (2007). Fingerprints recognition using minutiae extraction: a fuzzy approach. $14^{\text {th }}$ International Conference on Image Analysis and Processing, 229-234. https://doi.org/10.1109/ICIAP.2007.4362784

[8] Bartunek, J. S., Nilsson, M., Nordberg, J., Claesson, I. (2006). Neural network based minutiae extraction from skeletonized fingerprints. IEEE Region 10 Conference TENCON, 1-4. https://doi.org/10.1109/TENCON.2006.344104

[9] Sagar, V. K. \& Alex, K. J. B. (1999). Hybrid fuzzy logic and neural network model for fingerprint minutiae extraction. International Joint Conference on Neural Networks IJCNN'99, 5, 3255-3259. https://doi.org/10.1109//JCNN.1999.836178

[10] Gao, X., Chen, X., Cao, J., Deng, Z., Liu, C., \& Feng, J. (2010). A novel method of fingerprint minutiae extraction based on Gabor phase. $17^{\text {th }}$ IEEE International Conference on Image Processing (ICIP), 3077-3080. https://doi.org/10.1109/ICIP. 2010.5648893

[11] Zhang, X., Chen, C., Ni, L., \& Zhang, J. (2011). Fingerprint minutia extraction algorithm based on DSP. International Conference on Electronic \& Mechanical Engineering and Information Technology, 9, 4805-4808. https://doi.org/10.1109/EMEIT.2011.6024114

[12] Karungaru, S., Fukuda, K., Fukumi, M., Akamatsu, N. (2008). Classification of fingerprint images into individual classes using neural networks. $34^{\text {th }}$ Annual Conference of IEEE Industrial Electronics, IECON, 1857-1862. https://doi.org/10.1109/IECON.2008.4758238

[13] Lopez, M. \& Canto, E. (2008). FPGA implementation of a minutiae extraction fingerprint algorithm. IEEE International Symposium on Industrial Electronics (ISIE), 6, 1920-1925. https://doi.org/10.1109/ISIE.2008.4676920
[14] Vaikole, S., Sawarkar, S. D., Hivrale, S., \& Sharma, T. (2009). Minutiae feature extraction from fingerprint images. IEEE International Advance Computing Conference (IACC), 691-696. https://doi.org/10.1109/IADCC.2009.4809096

[15] Sudiro, S. A., Paindavoine, M., Yuwono, R. T., \& Etp, L. (2008). Performance evaluation of simple fingerprint minutiae extraction algorithm using crossing number on valley structure. International Conference on Innovations in Information Technology, 623-627. https://doi.org/10.1109/INNOVATIONS.2008.4781731

[16] Bhargava, N., Bhargava, R., Narooka, P., \& Cotia, M. (2012). Fingerprint recognition using minutia matching. International Journal of Computer Trends and Technology, 3(4), 641-643.

[17] Kekre, H. B. \& Bharadi, V. A. (2009). Fingerprints core point detection using orientation field. International Conference on Advances in Computing, Control \& Telecommunication Technologies, 150-152. https://doi.org/10.1109/ACT.2009.45

[18] Julasayvake, A. \& Choomchuay, S. (2007). An algorithm for fingerprint core point detection. $9^{\text {th }}$ International Signal Processing and Its Applications (ISSPA), 1-4. https://doi.org/10.1109/ISSPA.2007.4555384

[19] Ross, A., Jain A., \& Reisman, J. A. (2003). Hybrid fingerprint matcher. Pattern Recognition, 36, 1661-1673. https://doi.org/10.1016/S0031-3203(02)00349-7

[20] Tico, M. \& Kuosmanen, P. (2003). Fingerprint matching using an orientation-based minutia descriptor. IEEE Transactions on Pattern Analysis and Machine Intelligence, 25, 1009-1014. https://doi.org/10.1109/TPAMI.2003.1217604

[21] Hong, L., Wan, Y., \& Jain, A. K. (1988). Fingerprint image enhancement: Algorithm and performance evaluation. IEEE Transactions on Pattern Analysis and Machine Intelligence, 20, 777-789. https://doi.org/10.1109/34.709565

[22] Bazen, A. M. \& Gerez, S. H. (2002). Systematic methods for the computation of the directional fields and singular points of fingerprints. IEEE Transactions on Pattern Analysis and Machine Intelligence, 24, 905-919. https://doi.org/10.1109/TPAMI.2002.1017618

[23] Yang, J., Liu, L., Jiang, T., \& Fan, Y. (2003). A modified Gabor filter design method for fingerprint image enhancement. Pattern Recognition Letter, 24, 1805-1817. https://doi.org/10.1016/S0167-8655(03)00005-9

[24] Cheng, J. \& Tian, J. (2002). Fingerprint enhancement with dyadic scale space. $16^{\text {th }}$ International Conference on Pattern Recognition, 25, 1273-1284. https://doi.org/10.1109/ICPR.2002.1044651

[25] Wang, Y., Hu, J., \& Schroder, H. (2005). A gradient based weighted averaging method for estimation of fingerprint orientation fields. Proc. International Conference on Digital Image Computing: Techniques and Applications, 195-202. https://doi.org/10.1109/DICTA.2005.1578127

[26] http://homepages.inf.ed.ac.uk/rbf/HIPR2/sobel.htm

[27] Bartunek, J. S. (2005). Minutiae Extraction from fingerprint with neural network and minutiae based fingerprint verification. MSc Thesis, Teknologie Magisterexamen i Elektroteknik.

[28] Karaboga, D., Gorkemli, B., Ozturk, C. et al. (2014). A comprehensive survey: artificial bee colony (ABC) algorithm and applications. Artificial Intelligence Rev., 42(21). https://doi.org/10.1007/s10462-012-9328-0

[29] Specht, D. F. (1991). A General Regression Neural Network. IEEE transactions on neural networks, 2(6), 568576. https://doi.org/10.1109/72.97934

[30] Çam, Z. G., Çimen, S., \& Sedef, H. (2016). Generalized Regression Neural Network Based Efficient Memristor Modeling. The $10^{\text {th }}$ International Conference on Intelligent Systems and Control (ISCO), 1-4. 
[31] Maio, D., Maltoni, D., Cappelli, R., Wayman, J. L., \& Jain A. K. (2002). FVC2000: Fingerprint Verification Competition. IEEE Transactions on Pattern Analysis and Machine Intelligence, 24, 402-412. https://doi.org/10.1109/34.990140

[32] Batra, D., Singhal, G., \& Chaudhury, S. (2004). Gabor filter based fingerprint classification using support vector machines. Proceedings of the IEEE in India Annual Conference, (INDICON), 256-261. https://doi.org/10.1109/INDICO.2004.1497751

[33] Bei, S., Wusheng, L., Liebo, D., \& Qin, L. (2016). A fingerprint identification algorithm based on local minutiae topological property. IEEE International Conference on Data Science in Cyberspace (DSC), 694-697. https://doi.org/10.1109/DSC.2016.103

[34] Chavan, S., Mundada, P., \& Pal, D. (2015). Fingerprint authentication using Gabor filter based matching algorithm. International Conference on Technologies for Sustainable Development (ICTSD), 1-6. https://doi.org/10.1109/ICTSD.2015.7095910

[35] Chen, T. (2006). A novelty automatic fingerprint matching system. Intelligent Computing in Signal Processing and Pattern Recognition, 100-111. https://doi.org/10.1007/978-3-540-37258-5_11

[36] Jiang, X. \& Yau, W. Y. (2000). Fingerprint minutiae matching based on the local and global structures. International Conference on Pattern Recognition (ICPR), 6038-6041.

[37] Conti, V., Militello, C., Vitabile, S., \& Sorbello, F. (2010) Introducing pseudo-singularity points for efficient fingerprints classification and recognition. International Conference on Complex, Intelligent and Software Intensive Systems (CISIS), 368-375. https://doi.org/10.1109/CISIS.2010.134

[38] Bei, S., Wusheng, L., Liebo, D., \& Qin, L. (2016). A fingerprint identification algorithm based on local minutiae topological property. IEEE International Conference on Data Science in Cyberspace (DSC), 694-697. https://doi.org/10.1109/DSC.2016.103

[39] Xu, H. \& Veldhuis, R. N. (2010). Spectral minutiae representations for fingerprint recognition. Sixth International Conference on Intelligent Information Hiding and Multimedia Signal Processing (IIH-MSP), 341-345. https://doi.org/10.1109/IIHMSP.2010.90

[40] Gottschlich, C. \& Huckemann, S. (2014). Separating the real from the synthetic: Minutiae histograms as fingerprints of fingerprints. IET Biometrics, 3(4), 291-301. https://doi.org/10.1049/iet-bmt.2013.0065

\section{Contact information:}

\section{Dr. Nihan KAHRAMAN,}

Yildiz Technical University

Department of Electronics and Communications Engineering

34349 Beşiktaş - İstanbul / TURKEY

nihankahraman.yildiz@gmail.com

\section{Zehra Gulru CAM TASKIRAN,}

Yildiz Technical University

Department of Electronics and Communications Engineering

34349 Beşiktaş - İstanbul / TURKEY

\section{Murat TASKIRAN,}

Yildiz Technical University

Department of Electronics and Communications Engineering

34349 Beşiktaş - İstanbul / TURKEY 